\title{
HIKBI AT HALAKHAK SA BAGONG KADAWYAN: SIPAT-SURI SA KARANASAN NG MGA GURO SA PAGWAWASTO NG AWTPUT NG MGA MAG-AARAL
}

\author{
Dominic Patric G. Galdonez, PhD. \\ Philippine Science High School-Ilocos Region Campus
}

Article DOI: https://doi.org/10.36713/epra8864 DOI No: 10.36713/epra8864

\begin{abstract}
Abstrak
Maraming pagbabagong nagaganap dahil sa pandemyang kinahaharap ng mundo. Ang mga paaralan ang isa sa mga lubos na naapektuhan. Ipinahinto ang tradisyonal na pamamaraan ng pag-aaral at niyakap ang bagong kadawyan o anyo ng pagtuturo at pagkatuto. Isa sa mga problemang patuloy na kinahaharap ng mga guro ay ang pagwawasto ng mga awtput na ipinapasa ng mga mag-aaral. Nag-iisip ng iba't ibang gawain ang mga guro para lamang matugunan ang problemang ito. Kaya sa pag-aaral, inalam at sinuri ang mga makabuluhang karanasan ng mga guro sa pagwawasto ng mga awtput ng mga mag-aaral sa bagong kadawyan. Kuwalitatibong lapit ng pananaliksik ang ginamit sa pag-aaral. Ginamit ang naratibong disenyo (Gudmundsdottir, 2001) para dalumatin ang mga datos. Sa pagsusuri ng mga datos, napalitaw ang mga Hikbi (isyung kinahaharap) at Halakhak (estratehiyang ginagamit) ng mga guro sa pagwawasto ng mga awtput. Sa Hikbi, nabuo ang tatlong isyu bilang tema: pagkokontrol ng nilalaman ng mga awtput; pagpapasa ng mga awtput; at a paggamit ng mga online platform. Sa punto naman ng Halakhak, natukoy ang limang tema: paglalaan ng pahinga sa sarili; pagbibigay ng ekstensiyon; pakikipag-ugnayan sa mga magulang; paggamit ng iba't ibang plataporma; at pagbabahagi ng mga kagamitang pampagkatuto. Batay sa mga natuklasan, napansin na marami pa ring suliraning kinahaharap ang guro sa pagwawasto ng awtput ng mag-aaral gaya ng ipinatutupad na kurikulum; pamamaraan sa pagtataya at pagtatasa; kagamitang pampagtuturo; at sapat na kaalaman sa mga online platform na ginagamit. Likas ding mapamaraan ang mga guro (Asiegbu at Okpala, 2019). Marami silang mga estratehiyang ginagamit para masolusyonan ang problema. Ang mga ito ay nakatulong nang malaki sa mga guro para maibsan at maiwasan ang mga problema sa pagwawasto ng awtput ng mga mag-aaral.

Susing salita: Hikbi at Halakhak, Bagong Kadawyan, at Pagwawasto
\end{abstract}

\section{INTRODUKSIYON}

Sa panahon ng pandemya, maraming mga suliranin ang hinaharap ng sangkatauhan dahil sa pagbabago ng mga gawaing ating nakasanayan sa pang araw-araw na pamumuhay. Sinusubok ng pandemya ang katatagan ng mga tao. Marami ang naapektuhan at patuloy na naghihirap dahilan nang kakapusan at kawalan. Isa sa mga naapektuhang gawain ng tao ay ang pagtuturo at pagkatuto. Isa ang bansang Pilipinas sa lubhang naapektuhan ng pandemya, maraming mga paaralan at institusyon ang nagsara (Engzell, Frey, at Verhagen, 2020) para maibsan at maagapan ang lalong paglala ng mga nagkakasakit ng CoVid-19. Ngunit sa kabila nito, ang proseso ng mga paaralan ay kailangan pa ring magpatuloy (Esposito, Cotugno, at Principi, 2021). Kaya, ang mga paaralan ay nag-isip ng mga bagong estratehiya at pamamaraan para maisakatuparan pa rin ang mga layunin ng pagtuturo at pagkatuto. Dahil dito, ang lahat ay nakasalalay na sa kanilang mga gadget at internet (Brooks at Gierdowski, 2021) upang magawa ang mga bagay-bagay na nais sana nilang gawin sa labas ng bahay. Isa sa mga nakadepende sa mga kagamitang ito ay ang mga guro at mag-aaral para makapagturo at matuto ngunit, tulad ng ibang gawain, ay hindi ganoon kadaling makakuha, makabili, at gawin ang mga ito sapagkat maraming hadlang at problemang $(\mathrm{He}, \mathrm{Zhan}$, at $\mathrm{Li}$, 2021) hinaharap sa tinatawag nating remote na pagtuturo at pagkatuto. Ang remote na pagtuturo at pagkatuto o online learning ang isa malawakang pamamaraan na isinakatuparan at isinagawa ng mga paaralan para matugunan ang kahingiang magpatuloy pa rin ang pag-aaral sa kabila ng nararanasang pandemya. Ayon kay Nguyen (2015), epektibo ang online learning at maaaring mas maging epektibo pa kaysa sa tradisyonal na pagtuturo. Ngunit, hindi maikakaila na may mga problema pa rin sa remote learning, lalo na't bago pa lamang ito sa karamihan (Rotas at Kahapay, 2020). Ang pangunahing problema sa online learning ay ang pag-develop ng sense of community (Sun at Chen, 2016). Dahil sa 
birtuwal ang gawi ng pagpapatupad ng pagtuturo at pagkatuto, hindi nakikita nang personal ng mag-aaral ang kaniyang mga kapwa mag-aaral kaya marami sa kanila ang hindi makita ng iba ang essensiya ng konseptong ito dahil hindi nila maramdaman ang komunidad na nais nila. Sa pagtuturo naman, kinakailangan ng malaking kaukulang pinansiyal ng paaralan para matulungan nila ang mga guro at magaaral na nangangailangan ng mga kagamitan sa pagtuturo at pagkatuto (Mahmood, 2020).

Sa simula pa lang ay marami nang naging pangamba ang mga guro, estudyante at mga magulang sa bagong paraan ng pagtuturo ngayong panahon ng pandemya ngunit ito pa rin ay isinulong upang hindi tumigil ang mga mag-aaral sa kanilang pag-aaral. Ang mga ibinibigay na gawaing pagtatasa at pagtataya ay isa sa mga pinakakontrobersiyal na paksa ng mga administrador ng paaralan, guro, at iba pang mga kasapi ng komunidad (Tuscano, 2020). Dahil dito, ang mga guro ay patuloy na nag-iisip ng mga posibleng pamamaraan para maisakatuparan at maiwasan ang ganitong mga kontrobersiya. Nagpatupad ang mga guro ng mga formative at alternative na pagsusulit para matugunan ang pangangailangan na tayahin at tasahin ang kaalamang natatamo ng bata sa bagong anyo ng edukasyon at makapagbigay ng mga kaukulang tugon na magsisilbing isang anyo na rin ng pagkatuto sa bahagi ng mga mag-aaral (Renaissance.com, 2020). Ang pagwawasto sa mga pagsusulit, ang isa sa mga malalaking gampanin at problemang kinaharap at kinahaharap ng guro kapag pagtatasa at pagtataya ang nagiging usapin lalo na aspekto ng academic dishonesty (Guangul, Suhail, Khalit, at Khidir, 2020). Sa buong mundo, ang suliraning ito ang lalong ikinababahala ng mga paaralan (Finn at Frone, 2004; San Jose, 2021). Ang mga guro ay lalong nahihirapan sa kanilang sitwasyon sa pagwawasto ng mga awtput dahil sa mga konseptong ito. Imbes na magpatuloy lang sa pagwawasto ng mga awtput ay iniintindi nila ang mga bagay-bagay para maging patas at pantay ang pagbibigay ng marka sa mga mag-aaral. Maraming mga midyum at plataporma ang ginagamit ng mga guro para lamang matugunan ang usaping ito. Napansin na maraming kuwento mula sa mga guro (Schwarz, 2010) ang makukuha at malalaman sa konsepto ng pagwawasto ng awtput ng mga magaaral at marapat na makalap at matuklasan ang mga ito upang maging batayan sa anomang tuntunin sa mga institusyon (Hinnant-Crawford, 2016). Kaya sa pag-aaral na ito, sinikap na inalam at tinukoy ang karanasan ng mga guro sa pagwawasto ng mga awtput ng mga mag-aaral sa kabila ng kasalukuyang sistema ng edukasyon sa bansa. Mahalagang tukuyin ang mga karanasang ito para matugunan ang mga kailanganin at mapabuti ang mga polisiya at patakarang ipinatutupad bilang tugon sa krisis na dulot ng pandemya at mapabuti ang kalagayan ng mga guro sa mga kinahaharap na problema sa pagwawasto ng awtput ng mga mag-aaral.

\section{LAYUNIN NG PAG-AARAL}

Ang pag-aaral na ito ay nagtangkang alamin at suriin ang karanasan ng mga guro ng Philippine Science High School-Ilocos Region Campus sa panuruang taong 2020-2021 sa pagwawasto ng mga pagsusulit sa bagong kadawyan. Ispesipikong dinalumat din ang sumusunod: makalap at malaman ang karanasan ng mga guro sa pagwawasto ng mga pormatibo at alternatibong pagsusulit; makilatis ang mga estratehiya ng mga guro sa pagwawasto ng mga pormatibo at alternatibong pagsusulit; at makabuo ng mga temang bubuod sa mga karanasan ng mga guro.

\section{METODOLOHIYA}

\section{Disenyo ng Pag-aaral}

Ang pag-aaral na ito ay gumamit ng kuwalitatibong lapit ng pananaliksik. Isinakatuparan ang naratibong disenyo ng pananaliksik. Ang naratibong disenyo ng pananaliksik ay isang pagaaral sa karanasan ng tao sa mundo. Ang mga mananaliksik sa ganitong disenyo ay nangangalap ng mga kuwento at nagsusulat ng mga naratibo batay sa karanasan (Gudmundsdottir, 2001). Marami nang gumagamit sa ganitong disenyo ng pananaliksik lalong-lalo na ang mga guro, dahilan na likas sa kanila ang pagiging makuwento, mapabuhay man nila o sa lipunang kanilang ginagalawan (Connelly at Clandinin, 1990).

\section{Populasyon at Sampling}

Walong (8) guro ang naging kasangkot ng pag-aaral. Ang mga kasangkot ay mga guro na (1) nagtuturo sa Philippine Science High School-Ilocos Region Campus; (2) nakapagturo sa panuruang taong 2020-2021; (3) nasa dalawa o higit pang taon sa serbisyo; (4) at may karanasan sa pagwawasto ng awtput ng mga mag-aaral sa panahon ng implementasyon ng remote na pagtuturo at pagkatuto.. Gumamit ang mananaliksik ng purposive sampling sa pagtukoy ng mga kasangkot sa pag-aaral dahil may kaukulang katangiang taglay ang mga kasangkot na natukoy ng mananaliksik na makatutulong para matugunan ang layunin ng pagaaral (Palys, 2008).

\section{Instrumento ng Pag-aaral}

Para matukoy ang demograpikong pagkakakilanlan ng mga guro, bumuo ang mananaliksik ng talatanungan gamit ang Google Forms. Gumamit din ang mananaliksik ng isang researcher-made interview guide para makalap ang makabuluhang karanasan ng mga guro sa pagwawasto ng mg awtput ng mga mag-aaral. Ipinataya ang nilalaman ng instrumento sa tatlong guro ng Philippine Science High School-Ilocos 
Region Campus at napatunayang ito'y katanggaptanggap para gamitin sa pag-aaral.

\section{Paraan ng pangangalap at pagsusuri ng mga Datos}

Nagpamudmod ng survey questionnaire sa mga guro gamit ang Google Forms at dito inalam ang demograpikong pagkakakilanlan ng mga gurong kasangkot sa pag-aaral. Bago ang pagsasagawa ng pakikipanayam ay ipinakilala muna ng mananaliksik ang kaniyang sarili at ang tunguhin ng pakikipanayam. Humingi ang mananaliksik ng pahintulot sa mga kinapanayam para sa rekording. Ipinaliwanag din ang kumpedensiyalidad ng mga datos na nakalap.

Ang mga nakalap na datos sa pakikipanayam ay itinranskrayb at nilagyan ng mga kowd para matukoy ang mga kategorya at tema na ginamit para matugunan ang mga tanongpananaliksik. Sa pagsusuri ng mga nakalap na datos, ginamit ng mananaliksik ang pagsusuring cool at warm. Ayon kina de Guzman at Tan (2007) ang pagsusuring cool ay natranskrayb na datos na patuloy na binabasa para masuri at makita ang mga makabuluhang pahayag mula sa mga guro sa naging karanasan nila sa pagwawasto ng mga pagsusulit. Ang mga makabuluhang pahayag mula sa mga karanasan ay pagsasama-samahin para makabuo ng mga kategorya na gagamitin sa pagbubuo ng mga tema. Sa pagsusuring warm naman, ang mga natukoy na kategorya ay gagamitin sa tematikong pagsusuri sa pamamagitan ng pagtukoy sa pagkakapareho at ugnayan ng mga ito upang makabuo ng mga tema. Para matugunan ang kawastuhan ng mga resulta, ipinataya at ipinatasa ang mga nabuong tema sa pamamagitan ng critical friend na teknik.

\section{RESULTA AT PAGTALAKAY}

Sa pag-aaral, nakalap at nalaman ang karanasan ng walong uro ng Philippine Science High School-Ilocos Region Campus sa pagwawasto ng awtput ng mga mag-aaral. Ang mga gurong naging bahagi ng pag-aaral ay may pagkakakilanlan: lima ang lalaki at tatlo ang babae; lima sa kanila ay nagtamo ng digri sa masterado at tatlo naman ang kasalukuyang kumukuha ng masterado; apat sa kanila ay nagtuturo ng mga asignatura sa Agham, Matematika, at Teknolohiya at apat naman na nagtuturo ng mga asignatura sa Humanidades; apat sa kanila ay nasa serbisyo sa loob ng dalawa hanggang apat na taon, apat naman ang lima hanggang sampung taon, at dalawa ang nakapaglingkod na ng labing-isa hanggang labinlimang taon.

Ang mga datos na nakalap ay sinuri at binigyan ng interpretasyon. Mula sa mga nasuring datos, Nakita ang sumusunod: Natuklasan ang mga Hikbi (Isyu sa mga karanasan ng mga guro) at Halakhak (Estratehiya ng mga guro) sa pagwawasto ng awtput ng mga mag-aaral.

\section{Mga Hikbi ng mga guro sa pagwawasto ng awtput} Makikita sa talahanayan 1 ang mga Hikbi na kinaharap ng mga guro sa pagwawasto ng awtput ng mga mag-aaral. Nahahati ito sa tatlong sentral na tema ang: isyu sa pagkokontrol ng nilalaman; isyu sa pagpapasa ng mga awtput; at isyu sa paggamit ng mga online platforms.

Talahanayan 1. Karanasan ng mga guro sa pagwawasto ng awtput ng mga mag-aaral (Hikbi)

\begin{tabular}{|c|c|c|}
\hline Mga Piling Kowd/Pahayag & Kategorya & Tema \\
\hline $\begin{array}{l}\text { - I prefer not to give volume of } \\
\text { requirements to students kasi ako } \\
\text { rin mahihirapan. } \\
\text { I chose only the most essential } \\
\text { learning competencies, with that, } \\
\text { I crafted formative and } \\
\text { alternative assessments. } \\
\text { I prefer only giving 5-item quiz } \\
\text { for easy checking. Marami akong } \\
\text { ginagawa ngayon kasi unlike } \\
\text { before. } \\
\text { Nililimita ko ang number ng } \\
\text { items at kung minsan ginagawa } \\
\text { ko na lang na multiple choice } \\
\text { kasi. } \\
\text { Dahil sa change ng curriculum, I } \\
\text { prefer to also change the way I } \\
\text { presented topics and assessments } \\
\text { to students. }\end{array}$ & $\begin{array}{l}\text { Kontrol sa Pagbibigay ng } \\
\text { Awtput }\end{array}$ & $\begin{array}{l}\text { Isyu sa Pagkokontrol sa } \\
\text { nilalaman ng awtput }\end{array}$ \\
\hline - $\quad$ May mga bata na hindi nagsa- & $\begin{array}{l}\text { Hindi nakapagpapasa ang mga } \\
\text { mag-aaral ng awtput }\end{array}$ & $\begin{array}{l}\text { Isyu sa Pagpapasa ng mga } \\
\text { awtput }\end{array}$ \\
\hline
\end{tabular}




\begin{tabular}{|c|c|c|}
\hline $\begin{array}{l}\text { submit ng kanilang mga awtput } \\
\text { sa tamang oras. } \\
\text { Medyo nakaka-stress dahil hindi } \\
\text { lahat ay nagpapasa on time. } \\
\text { - Nahirapan sa pagkumbising } \\
\text { ipasa ang awtput sa nakatakdang } \\
\text { panahon. }\end{array}$ & & \\
\hline $\begin{array}{l}\text { - Medyo mahirap ang online } \\
\text { checking kasi dapat matapos mo } \\
\text { ito. Babad ang mata sa computer } \\
\text { maghapon. } \\
\text { Paputol-putol ang pagwawasto } \\
\text { dahil masakit sa mata lalo na } \\
\text { kung mahaba ang screen time. } \\
\text { Mas mahirap mag-check ngayon } \\
\text { kumpara noong F2F, } \\
\text { nakakapagod yong pag scroll sa } \\
\text { screen tapos additonal source of } \\
\text { stress pa yong exposure sa } \\
\text { radiation. }\end{array}$ & $\begin{array}{l}\text { Mahabang eksposyur sa } \\
\text { kompyuter }\end{array}$ & \multirow[t]{3}{*}{$\begin{array}{l}\text { Isyu sa Paggamit ng mga } \\
\text { online platforms sa mga } \\
\text { awtput }\end{array}$} \\
\hline $\begin{array}{l}\text { - Minsan natatambakan ng } \\
\text { trabaho dahil nailipat sa ibang } \\
\text { araw ang dapat na tapos na dahil } \\
\text { sa technical problems. } \\
\text { Maraming problema gaya ng } \\
\text { sudden power interruption, Khub } \\
\text { maintenance, kawalan ng } \\
\text { internet dahil sa system upgrade } \\
\text { ng mga internet providers o dahil } \\
\text { sa malakas ang ulan o bagyo }\end{array}$ & Teknikal na problema & \\
\hline 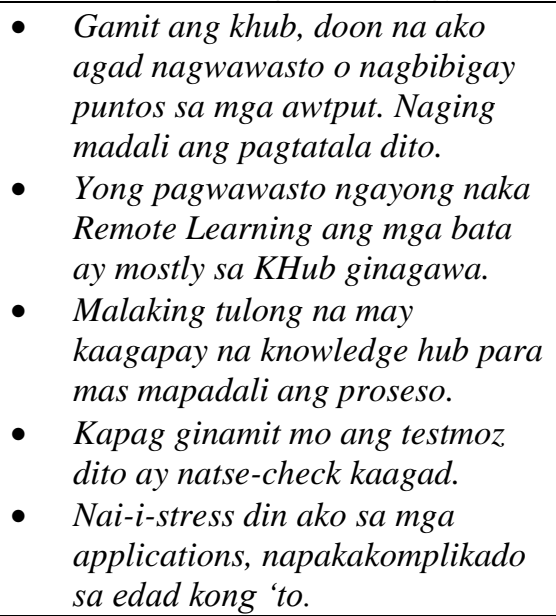 & $\begin{array}{l}\text { Kagamitan sa pagwawasto ng } \\
\text { mga awtput }\end{array}$ & \\
\hline
\end{tabular}

\section{Isyu sa pagkokontrol ng nilalaman ng awtput} Maraming pagbabago ang naganap at isinakatuparan para matugunan ang problemang kinahaharap ng bansa lalo na sa sistema ng edukasyon-kurikulum, pananaliksik, pagtataya, at pananaliksik (Huber at Helm, 2020).

Sa karanasan ng Philippine Science High School System, nagkaroon ito ng malawakang sa kanilang kurikulum para matugunan ang kahingian ng kasalukuyang sitwasyon. Maraming paksa ang tinanggal sa bawat asignatura; gayundin ang mga kasanayan o learning competencies na dapat matamo ng isang mag-aaral. Pinili lamang ang mga most essential learning competencies na ilalagay sa kurikulum. Napansin din ang paglilimita ng mga guro sa nilalaman ng kani-kanilang mga pagsusulit kung ikukumpara sa mga gawaing ipinapatupad nila sa tradisyonal na sistema ng edukasyon. Ayon sa kanila kaya ginagawa nila ito ay upang maibsan ang pagod 
sa pagwawasto ng mga ito at maiwasan ang sobrang eksposyur sa radiation na dala ng kompyuter.

Isa rin sa mga nakitang umuusbong na dahilan sa paglilimita ay ang pagdami ng trabaho ng isang guro (Jankowski, 2020) kung ihahambing ito sa tradisyonal na pamamaraan. Sa kaso ng PSHS-IRC, maraming workload ang naidagdag sa trabaho ng mga guro. Malaki rin ang ambag sa pagbabago at paglilimita sa mga awtput ay dahil sa kondisyon ng institusyon (Shavelson et al., 2010).

\section{Isyu sa pagpapasa ng mga awtput}

Kaakibat na sa pagtuturo at pagkatuto ang mga problema (Lodge, Kennedy, Lockyer, Arguel, at Pachman, 2018). Mahalaga sa pagtatasa at pagtataya ang pagbibigay ng tugon at marka sa mga awtput at pagusulit. Higit ding natututo ang mga mag-aaral na hindi gaanong nakasusunod sa paksa at ang mga mag-aaral naman na mabili matuto ay lalong nagaganyak (Semerci, 2007; Metin 2013). Ngunit, dahil sa kasalukuyang sitwasyon, hindi maikakaila na isa sa mga malalaking isyu na kinahaharap ng mga guro ay ang paghihintay at pagpapasa ng mga awtput mula sa mga mag-aaral. Hindi sila kaagad nakapagbibigay nang agarang tugon sa mga pagtataya o pagsusulit dahil sa walang ipinapasa ang mga mag-aaral. Naaapektuhan din ang ibang gawain ng mga guro dahil dito. Napansin din mula sa mga datos na nagdudulot din ng stress sa bahagi ng guro kapag hindi nakapagpapasa ang mga mag-aaral ng kani-kanilang mga awtput. Sinusugan ito ni Soriano (2021) sa kaniyang artikulo kung saan ang mga gurong nakapanayam niya ay nakaramdam ng stress at panghihinayang dahil hindi nagpapasa ang mga mag-aaral ng mga awtput o pangangailangan sa klase.

\section{Isyu sa paggamit ng mga online platforms}

Marami sa mga guro ang nanibago sa bagong sistema ng pagtuturo dulot ng pandemya
(Barron, Cobo, Munoz-Najar, at Ciarrusta, 2021) kaya kailangan nilang matutuhan ang mga bagaybagay na nauukol dito (Konig, Jager-Biela, at Glutsch, 2020) para maipagpatuloy pa rin ang pagtuturo. Mula sa mga kinapanayam, napansin na may mangilan-ngilang nahihirapan sa paggamit ng mga online platform at minsan ay nagiging dagdag na rin ito sa kanilang problema. May ilan namang guro na nagagaanan sa pagwawasto ng mga awtput dahil sa mga application o software na kanilang ginagamit ngunit nakararanas pa rin sila ng mga problema sa paggamit ng mga ito lalong-lalo na kung nawawalan sila ng koneksiyon sa internet, may sakuna, o di kaya'y walang kuryente. Ang mga isyung naturan ay nakapagdudulot ng kabiguan sa bahagi ng mga guro dahil hindi nila nagagawa ang pagwawasto sa takdang panahon na kanilang inilaan. Natatambakan din sila ng kanilang mga iwawastong awtput kapag nararanasan nila ang mga problemang ito. Nagiging problema rin ng guro ang mahabang pagbabad sa kompyuter kapag ito ay nagwawasto ng mga awtput. Nababahala sila sa maaaring masamang dulot ng radiation sa kani-kanilang mga kalusugan.

\section{Mga Halakhak ng mga guro sa pagwawasto ng awtput}

Ang mga guro ay likas na mapamaraan (Asiegbu at Okpala, 2019) kaya madali lanag nilang nasosolusyonan mga suliraning kinahaharap lalonglalo na sa aspekto ng pagtuturo at pagkatuto. Mapapansin sa Talahanayan 2 ang mga Halakhak ng mga guro sa pagwawasto ng awtput ng mga magaaral. Ang mga ito ang naging estratehiya o pamamaraan nila para matugunan ang mga problemang kinaharap nila sa pagwawasto ng mga awtput ng mag-aaral. Nahanay ang mga ito limang tema: paglalaan ng pahinga sa sarili; pagbibigay ng ekstensiyon; pakikipag-ugnayan sa mga magulang; paggamit ng iba't ibang plataporma; at pagbabahagi ng mga kagamitang pampagkatuto.

Talahanayan 2. Estratehiya ng mga guro sa pagwawasto ng awtput ng mga mag-aaral (Halakhak)

\begin{tabular}{|c|c|}
\hline Mga Piling Kowd/Pahayag & 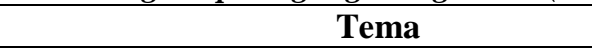 \\
\hline $\begin{array}{l}\text { - Sinisigurado ko na may break time ako para } \\
\text { hindi masyadong mahaba ang aking screen } \\
\text { time. } \\
\text { - Nilimit ko radiation exposure kapag } \\
\text { magcheck, most of the time. } \\
\text { Kailangang ipahinga ang mga mata para } \\
\text { may lakas pa sa susunod na mga awtput na } \\
\text { itse-check. }\end{array}$ & Paglalaan ng pahinga sa sarili \\
\hline $\begin{array}{l}\text { - Hinahabaan ko na lang ang aking pasensya } \\
\text { at mahinahon ko silang pinapadalhan ng } \\
\text { mensahe via email o messenger } \\
\text { - } \quad \text { I-reschedule na lamang ang mga } \\
\text { nakaplanong gawain } \\
\text { - Pagbibigay ng extension o palugit sa }\end{array}$ & Pagbibigay ng ekstensiyon sa mga palugit \\
\hline
\end{tabular}


pagpasa ng mga gawain.

- $\quad$ Totoong medyo nahirapan ang mga guro pero ang mahalaga ay nakatulong ito sa mga bata sa pagbibigay ng extension.

- Bigyan nang sapat na panahon para magawa ng mga bata ang kanilang $m g a$ awtput.

- Kinokontak ko na lang ang kanilang adviser at magulang para humingi ng tulong

- Pagbibigay ng Academic Performance Form para ipaalam ang kanilang performance at maipagbigay alam din ito sa mga magulang para sa tamang gabay.

- $\quad$ Gumamit din ng ibang platform para sa pagpapaalala.

Pakikipag-ugnayan sa mga magulang

Pagbibigay ng intervention period para bigyan klaro ang mga suliranin.

- Ginagamit ko 'yong platform na nakita ko sa isang webinar na sinalihan ko.

- A lot of strategies and platforms in addressing this concern where being taught to us in a webinar that I attended, I used those.

- Pagpapahiram ng mga laptop, desktop, at pagbibigay ng load allowance para sa mga walang kakayahan na mag-remote learning.

- I shared some of my online platforms to them like personal canva accounts, especially those who are in need.

Paggamit ng iba't ibang plataporma

Pagbabahagi ng mga kagamitang pampagkatuto

\section{Paglalaan ng pahinga sa sarili}

Mahalaga para sa mga gurong kinapanayam ang pagpapahinga. Kailangan nila ito upang magampanan ang iba pa nilang mga tungkulin bilang guro. Ang sapat na pahinga ay mahalaga para magawa ang mga gawain nang epektibo at mahusay (Veldhoen, 2016). Ang labis na eksposyur din sa radiation ang isa sa mga ikinababahala ng mga guro kaya nagkakaroon sila nang panandaliang pagpapahinga para ma-relax ang kani-kanilang mga mata.

\section{Pagbibigay ng ekstensiyon sa mga palugit}

Marami sa mga mag-aaral ang hindi nakapagpapasa ng kani-kanilang mga awtput dahil sa iba't ibang dahilan o salik (Tani, Dalzell, Ehambaranathu, Murugasu, at Steele, 2019). Dahil sa kagustuhan ng mga guro na lalong matuto ang mga mag-aaral at hindi maiiwan ay nagbibigay ang mga guro ng mga ekstensiyon sa mga itinakdang palugit para mabigyan pa ng sapat na panahon ang mga magaaral na gawin ang mga pangangailangan nila sa loob ng klase. Nagsisilbing tulong na rin ng mga guro ang pagbibigay ng ekstensiyon sa mga mag-aaral. Batid ng mga guro ang iba't ibang salik na maaaring nakaapekto sa hindi pagpapapasa ng mag-aaral.

\section{Pakikipag-ugnayan sa mga magulang}

Malaki ang gampanin ng mga magulang sa proseso ng pagtuturo at pagkatuto ng mga mag-aaral (Ceka at Murati, 2016) lalong-lalo na sa kasalukuyang sistema ng edukasyon. Nakasalalay sa mga magulang ang pagganyak para gawin ng mga mag-aaral ang kanilang mga awtput o pangangailangan sa klase. Kapag batid na ng guro na marami ng liban ang mag-aaral o di kaya'y natatambakan na ito ng mga kinakailangang ipasa ay nakikipag-ugnayan ang mga guro sa mga magulang ng bata upang kausapin at ipaalam ang sitwasyon ng mag-aaral. Nagiging kaagapay o katuwang ng guro ang mga magulang para gabayan at paalalahanan ang mga mag-aaral sa kani-kanilang mga responsibilidad sa proseso ng pagtuturo at pagkatuto. 


\section{Paggamit ng iba't ibang plataporma}

Buhat sa mga webinar at palihan na dinaluhan ng mga guro, natuto at nakakalap sila ng mga idea na maaari nilang gamitin para matugunan ang problema nila sa pagwawasto ng awtput ng mga mag-aaral. Naglalaan din sila ng mga intervention period para masagot at mabigyang-linaw ang mga paksa o konsepto na hindi gaanong naintindihan o naunawaan ng mag-aaral.

\section{Pagbabahagi ng kagamitang pampagkatuto}

Hindi pare-pareho ang antas ng pamumuhay ng mga mag-aaral, kaya ang ilan ay nahihirapan sa bagong anyo ng edukasyon sa bansa. Marami ang hindi nakakakayang bumili ng mga kagamitan para makapagpatuloy sa pag-aaral. Mahirap sa kanila ang sitwasyong ito na madalas nagiging sanhi ng hindi nila pagpapasa o paggawa ng mga pangangailangan sa kani-kanilang mga asignatura. Mula sa mga nakapanayam, ibinabahagi nila ang ilan sa kanikanilang mga kagamitan sa mga mag-aaral para matulungan lamang ang mga ito na makapagpasa ng mga awtput at hindi sila maiwan sa proseso ng pagtuturo at pagkatuto. Nagbabahagi rin ang iba ng load allowance bilang tulong sa bayad ng internet na gagamitin ng mag-aaral sa pagdalo ng kaniyang mga klase at pagsagot na rin ng mga awtput o pagsusulit.

\section{KONGKLUSYON AT REKOMENDASYON}

Batay sa mga natuklasan, mapapansin na marami pa ring suliraning kinahaharap ang guro sa pagwawasto ng awtput ng mag-aaral gaya ng problema sa ipinatutupad na kurikulum; pamamaraan sa pagtataya at pagtatasa; mga kagamitang pampagtuturo; at sapat na kaalaman sa mga online platform na ginagamit para maipatupad ang kasalukuyang sistema ng edukasyon. Likas ding mapamaraan ang mga guro. Marami silang mga estratehiyang isinakatuparan para masolusyonan ang problema gaya ng paglalaan ng pahinga sa sarili; pagbibigay ng ekstensiyon sa mga palugit; pakikipagugnayan sa mga magulang; paggamit ng iba't ibang plataporma; at pagbabahagi ng mga kagamitang pampagkatuto. Ang mga ito ay nakatulong sa mga guro para maibsan at maiwasan ang mga problema ng guro sa pagwawasto ng awtput ng mga mag-aaral. Buhat sa mga nakitang resulta, iminumungkahi na patibayin ang mga polisiya na may kinalaman sa kurikulum, pagtatasa, at pagtataya; paglalaan ng sapat na pondo para sa mga kagamitang pampagtuturo; at training para sa mga guro upang lalong mapayabong ang kanilang kasanayan at kakayahan at makasabay sa bagong anyo ng pagtuturo at pagkatuto.

\section{SANGGUNIAN}

1. de Guzman, A., \& Tan, E. (2007). Understanding the essence of scholarship from the lived experiences of a select group of outstanding Filipino researchers. Educational Research Journal 22 (1), 49-68

2. Asiegbu, F., \& Okpala, J. (2019). Teacher Resourcefulness as a Key to Improving the use of Instructional Materials in Teaching and Learning of Mathematics in Primary Schools in Anambra State. Multidisciplinary Journal of Research and Development, 3(1).

3. Barron, M., Cobo, C., Munoz-Najar, A., \& Ciarrusta, I. (2021). The changing role of teachers and technologies amidst the COVID 19 pandemic: Key findings from a cross-country study. World Bank Blogs. Retrieved September 21, 2021, from https://blogs.worldbank.org/education/changingrole-teachers-and-technologies-amidst-covid-19pandemic-key-findings-cross.

4. Brooks, D., \& Gierdowski, D. (2021). Student Experiences with Technology in the Pandemic. EduCause Research.

5. Ceka, A., \& Murati, R. (2016). The Role of Parents in the Education of Children. Journal of Education and Practice, 7(5).

6. Connelly, M. F., \& Clandinin, J. D. (1990). Stories of experience and narrative inquiry. Educational Researcher, 19(5), 2-14.

7. de Guzman, A., \& Tan, E. (2007). Understanding the essence of scholarship from the lived experiences of select group of outstanding Filipino researchers. Educational Research Journal, 22(1), 49-68.

8. Engzell, P., Frey, A., \& Verhagen, M. D. (2020). Learning loss due to school closures during the COVID-19 https://doi.org/10.31235/osf.io/ve4z7

9. Esposito, S., Cotugno, N., \& Principi, N. (2021). Comprehensive and safe school strategy during COVID-19 pandemic. Italian Journal of Pediatrics, $\quad 47(1)$ https://doi.org/10.1186/s13052-021-00960-6

10. Guangul, F. M., Suhail, A. H., Khalit, M. I., \& Khidhir, B. A. (2020). Challenges of remote assessment in higher education in the context of COVID-19: A case study of middle east college. Educational Assessment, Evaluation and Accountability, 32(4), 519-535. https://doi.org/10.1007/s11092-020-09340-w

11. Gudmundsdottir, S. (2001). Narrative research on school practice. In V. Richardson (Ed.), Fourth handbook for research on teaching ( $p p$. 226-240). New York: MacMillan.

12. He, W., Zhang, Z. (J., \& Li, W. (2021). Information Technology Solutions, challenges, and suggestions for tackling the COVID-19 pandemic. International Journal of Information Management, $\quad 57, \quad 102287$. https://doi.org/10.1016/j.ijinfomgt.2020.102287

13. Hinnant-Crawford, B. (2016). Education Policy Influence Efficacy: Teacher Beliefs in their Ability to Change Education Policy. International Journal of Teacher Leadership, $7(2)$.

14. Huber, S. G., \& Helm, C. (2020). Covid-19 and schooling: Evaluation, assessment and accountability in times of crises-reacting quickly to explore key issues for policy, practice 
and research with the School Barometer. Educational Assessment, Evaluation and $\begin{array}{lr}\text { Accountability, } \quad 32(2), & 237-270 .\end{array}$ https://doi.org/10.1007/s11092-020-09322-y

15. Jankowski, N. A. (2020, August). Assessment during a crisis: Responding to a global pandemic.

16. Urbana, IL: University of Illinois and Indiana University, National Institute for Learning

17. König, J., Jäger-Biela, D. J., \& Glutsch, N. (2020). Adapting to online teaching during COVID-19 school closure: Teacher education and teacher competence effects among early career teachers in Germany. European Journal of Teacher Education, 43(4), 608-622. https://doi.org/10.1080/02619768.2020.1809650

18. Lodge, J. M., Kennedy, G., Lockyer, L., Arguel, A., \& Pachman, M. (2018). Understanding difficulties and resulting confusion in Learning: An integrative review. Frontiers in Education, 3. https://doi.org/10.3389/feduc.2018.00049

19. de Guzman, A., \& Tan, E. (2007). Understanding the essence of scholarship from the lived experiences of a select group of outstanding Filipino researchers. Educational Research Journal 22 (1), 49-68

20. Mahmood, S. (2020). Instructional strategies for online teaching in COVID-19 pandemic. Human Behavior \& Emerging Technologies, 1-5. https://doi.org/10.1002/hbe2.218

21. Metin, M. (2013). Teachers' Difficulties in Preparation and Implementation of performance Task. Educational Sciences: Theory and Practice. https://doi.org/10.12738/estp.2013.3.1452

22. Nguyen, T. (2015). The Effectiveness of online learning: Beyond no significant difference and future horizons. Journal of Information Technology Education: Research, 11(2), 309319.

23. Palys, T. (2008). Purposive sampling. In L. M. Given (Ed.) The Sage Encyclopedia of Qualitative Research Methods. (Vol.2). Sage: Los Angeles, pp. 697-8.

24. Rotas, E., \& Cahapay, M. (2020). Difficulties in Remote Learning: Voices of Philippine
University Students in the Wake of COVID-19 Crisis. Asian Journal of Distance Education, 15(2).

25. San Jose, A. (2021). Academic Integrity during COVID 19 Pandemic. A Student Perspective. GRIN Verlag. https://www.grin.com/documeny/1012391

26. Schwarz, G. (2001). Using teacher narrative research in teacher development. The Teacher Educator, $\quad 37(1), \quad 37-48$. https://doi.org/10.1080/08878730109555279

27. Soriano, L. (2021, January 24). Mga Guro Dismayado sa Hindi Pagpapasa ng requirements ng mga estudyante. The POST. Retrieved September 21, 2021, from https://thepost.net.ph/news/nation/mga-gurodismayado-sa-hindi-pagpapasa-ng-requirementsng-mga-estudyante/.

28. Sun, A. \& Chen, X. (2016). Online education and its effective practice: A research review. Journal of Information Technology Education: Research, 15, 157-190.

29. Tani, K., Dalzell, E., Ehambaranathan, N., Murugasu, S., \& Steele, A. (2019). Evaluation of factors affecting students' performance in tertiary education. Journal of Pedagogical Research, $3(2)$, $1-10$. https://doi.org/10.33902/jpr.2019252504

30. Tuscano, F. J. (2020, May 2). Reflections on assessments and grades at this time of the pandemic: An interview with teacherly. empowerED. Retrieved September 20, 2021, from https://francisjimtuscano.com/2020/05/02/reflecti ons-on-assessments-and-grades-at-this-time-ofthe-pandemic-an-interview-withteacherly/.Understanding assessment's "New normal". Renaissance. (2021, September 15). Retrieved September 20, 2021, from https://www.renaissance.com/2020/08/14/blogunderstanding-assessments-new-normal/.

31. Veldhoen, K. (2016, May 2). Rest: The antidote for discouragement. Edutopia. Retrieved September 21, 2021, from https://www.edutopia.org/blog/rest-the-antidotefor-discouragement-karine-veldhoen. 